\title{
Physics at the Tevatron Run II
}

\author{
D. Zieminska ${ }^{a}$ \\ ${ }^{a}$ Indiana University, Physics Department Bloomington, IN 47405, U.S.A
}

We present recent physics results from the Tevatron Collider experiments CDF and DØ.

\section{INTRODUCTION}

The Tevatron Collider Run II started in March 2002 and is expected to continue until the end of this decade. The Tevatron and the two detectors, CDF and DØ, have been performing well in 2004 , each experiment is collecting data at the rate of $\approx 10 \mathrm{pb}^{-1}$ per week. The total luminosity accumulated by August 2004 is $\approx 500 \mathrm{pb}^{-1}$ per detector. The rich physics program includes the production and precision measurement of properties of standard model (SM) objects, as well as searches for phenomena beyond standard model. In this brief review we focus on areas of most interest to the lattice community. We present new results on the top quark mass and their implication for the mass of the SM Higgs boson, on searches for the SM Higgs boson, on evidence for the $X(3872)$ state, on searches for pentaquarks, and on $b$ hadron properties. All Run II results presented here are preliminary.

\section{TOP QUARK MASS}

The experiments CDF and D $\varnothing$ published several direct measurements of the top quark pole mass, $M_{\text {top }}$, based on Run I data (1992-1996). The "lepton + jets" channel yields the most precise determination of $M_{\mathrm{top}}$. Recently, the DØ collaboration published a new measurement [1, based on a powerful analysis technique yielding greatly improved precision. The differential probability that the measured variables in any event correspond to the signal is calculated as a function of $M_{\mathrm{top}}$. The maximum in the product of the individual event probabilities provides the best estimate of $M_{\text {top }}$. The critical differences from previous analyses in the lepton + jets decay channel lie in the assignment of more weight to events that are well measured or more likely to correspond to $t \bar{t}$ signal, and the handling of the combinations of final-state objects (lepton, jets, and imbalance in transverse momentum) and their identification with top-quark decay products in an event. The new combined value for the top-quark mass from Run I is $M_{\text {top }}=178.0 \pm 4.3 \mathrm{GeV} / c^{2}$.

In Run II, both collaborations have been exploring several different techniques for $M_{\text {top }}$ measurements. The best single CDF result comes from a dynamic likelihood method (DLM). The method is similar to the technique used in Ref. [1]. The result is $M_{\text {top }}=177.8_{-5.0}^{+4.5}($ stat $) \pm$ 6.2 (syst) $\mathrm{GeV} / c^{2}$. The joint likelihood of the selected events is shown in Fig. 1] The Run II goal is a $1 \%$ uncertainty on $M_{\text {top }}$.

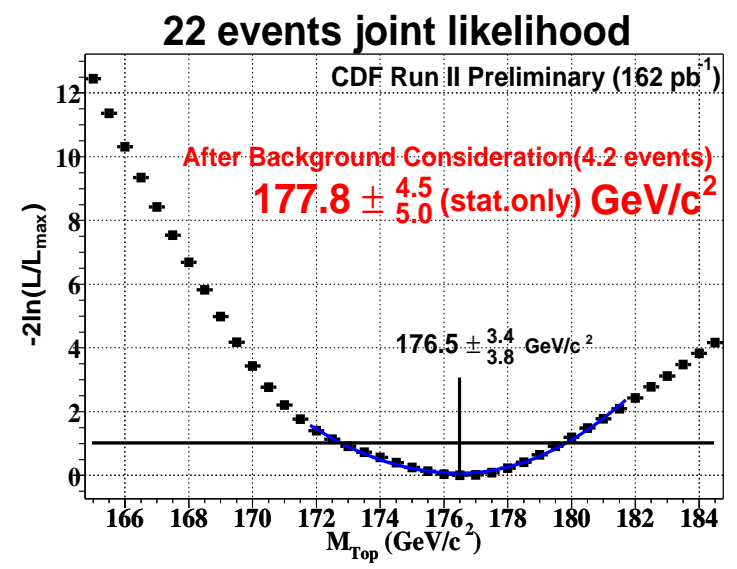

Figure 1. The joint likelihood of top candidates $(\mathrm{CDF})$. 


\section{SEARCH FOR SM HIGGS BOSON}

The constraints on the SM Higgs $(H)$ boson mass from published measurements, updated to include the new $\mathrm{D} \varnothing$ top mass measurement [1, are $M_{H}=117_{-45}^{+67} \mathrm{GeV} / c^{2}, M_{H}<251 \mathrm{GeV} / c^{2}$ at $95 \%$ C.L. The new most likely value of $M_{H}$ is above the experimentally excluded range, and sufficiently low for $H$ to be observed at the Tevatron.

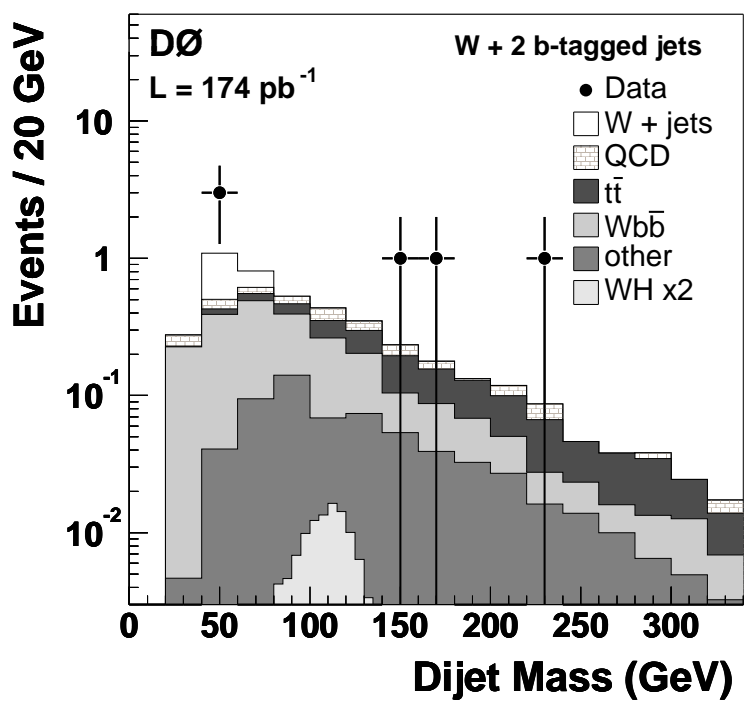

Figure 2. Distribution of the dijet invariant mass for $W+2 b$-tagged jets events, compared to the expectation $(\mathrm{D} \varnothing)$.

DØ has conducted a search for $H$ at $M_{H}<$ $140 \mathrm{GeV} / c^{2}$ in the production channel $p \bar{p} \rightarrow$ $W H \rightarrow e \nu b \bar{b}$. The experimental signature of $W H \rightarrow e \nu b \bar{b}$ is a final state with one high $p_{T}$ electron, two $b$ jets, and large missing transverse energy resulting from the undetected neutrino. The dominant backgrounds to $W H$ production are $W b \bar{b}, t \bar{t}$ and single-top production. The distribution of the dijet mass for events with two $b$-tagged jets is shown in Fig. 2 Also shown is the expected contribution (0.06 events) from the $b \bar{b}$ decay of a SM Higgs boson with $M_{H}=115$ $\mathrm{GeV} / c^{2}$. No events are observed in the dijet mass window of $85-135 \mathrm{GeV} / c^{2}$. DØ sets a limit on the cross section for $\sigma(p \bar{p} \rightarrow W H) \times B(H \rightarrow b \bar{b})$ of $9.0 \mathrm{pb}$ at the $95 \%$ C.L., for a $115 \mathrm{GeV} / \mathrm{c}^{2}$ Higgs boson. The results for mass points 105, 125, and $135 \mathrm{GeV} / \mathrm{c}^{2}$ are 11.0, 9.1 and $12.2 \mathrm{pb}$, respectively.

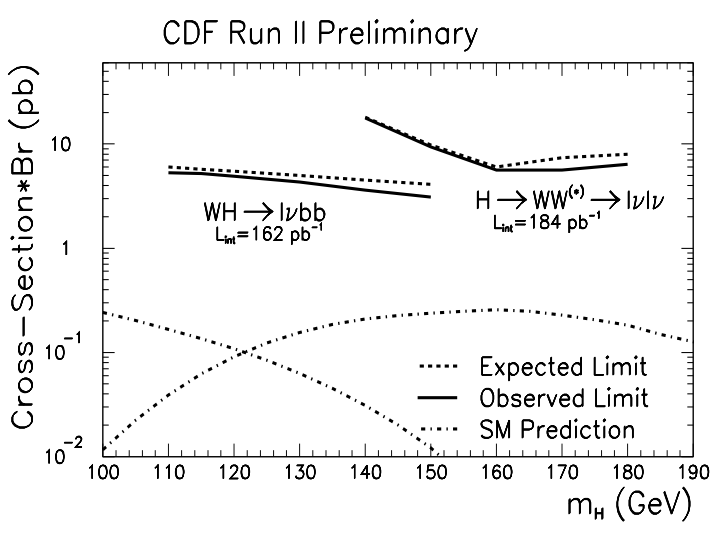

Figure 3. 95\% limits on the $H$ production (CDF).

CDF has done a similar search, allowing either an electron or a muon in the final state. Both groups have also searched for $H$ produced in gluon-gluon fusion, with subsequent decay to a pair of $W$ bosons. The CDF results for both channels are shown in Fig. 3

\section{THE STATE $\mathrm{X}(\mathbf{3 8 7 2})$}

The existence of the $X(3872)$ state discovered by the Belle Collaboration [2] has been confirmed in $p \bar{p}$ collisions by $\mathrm{CDF}$ [3] (see Fig. 4) and $\mathrm{D} \varnothing$ [4. It is still unclear whether this particle is a $c \bar{c}$ state, or a more complex object. When the data are separated according to production and decay variables, D $\varnothing$ finds no significant differences between the $X(3872)$ and the $c \bar{c}$ state $\psi(2 S)$. CDF has analysed the "lifetime" distribution of the $X(3872)$ events in order to quantify what fraction of this state arises from decay of $B$ hadrons, as opposed to those produced promptly. The authors find that for the selected samples $28.3 \pm 1.0($ stat $) \pm 0.7$ (syst)\% of $\psi(2 S)$ candidates are from $b$ decays, whereas 


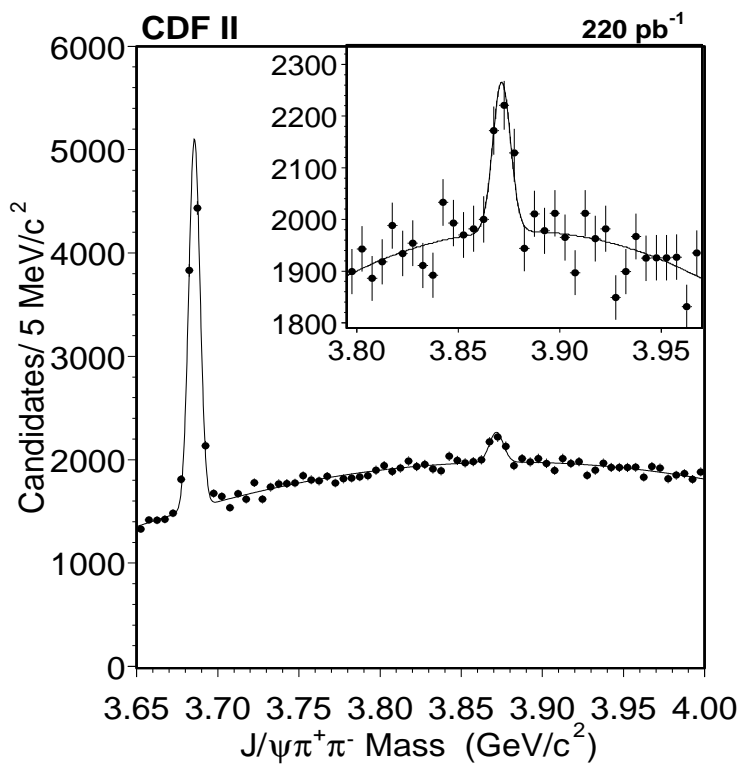

Figure 4. The $X(3872)$ signal $(\mathrm{CDF})$.

$16.1 \pm 4.9$ (stat) \pm 2.0 (syst) $\%$ of $X$ mesons arise from such decays.

\section{SEARCH FOR PENTAQUARKS}

Following reports of evidence for exotic baryons containing five quarks (pentaquarks), CDF has analysed its data for evidence of the following pentaquarks: $\Theta^{+}(u u d \bar{d} \bar{s})$, doubly strange states $\Xi_{3 / 2}$, charmed states $\Theta_{c}$, and, most recently, a state $(u d u s \bar{b})$, dubbed $R_{s}^{+}$, through its weak decay to $(J / \psi, p)$. With its excellent particle indentification and mass resolution, $\mathrm{CDF}$ has a unique capability to search for pentaquark states. The signals of known states: $\phi, \Lambda, \Lambda(1520), K^{*}, \Xi$, compare favorably with those provided by the authors of the pentaquark evidence. The group finds no evidence for pentaquark states, see Figs 5617. This can be interpreted as an indication that the pentaquark production in $p \bar{p}$ collisions is heavily suppressed compared to the conventional hadron production, or as an evidence against the existence of pentaquarks.

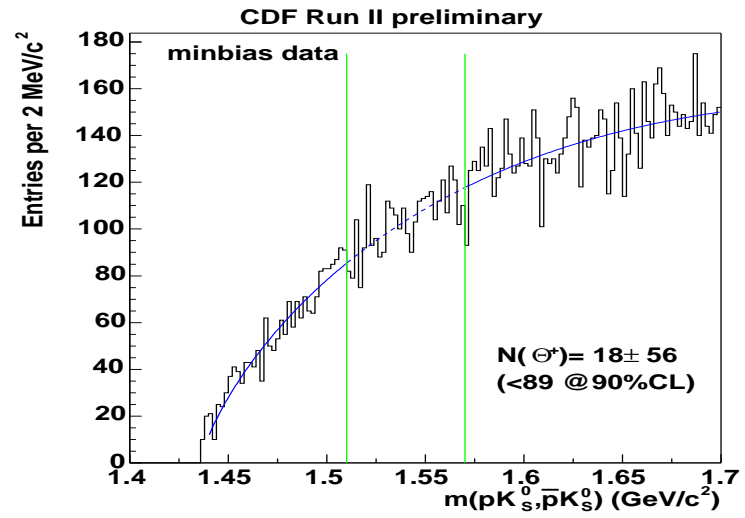

Figure 5. Invariant mass distribution of an identified proton and a $K_{s}^{0}$ candidate. $(\mathrm{CDF})$

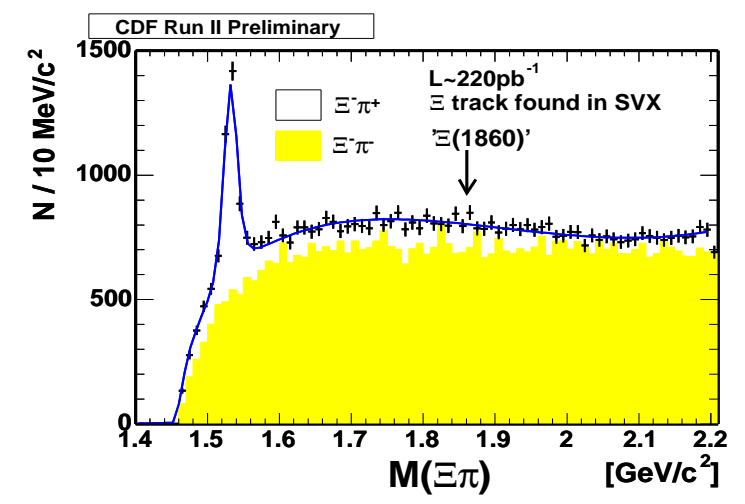

Figure 6. Invariant mass distribution of the $\left(\Xi^{-}, \pi^{+}\right)$system. (CDF)

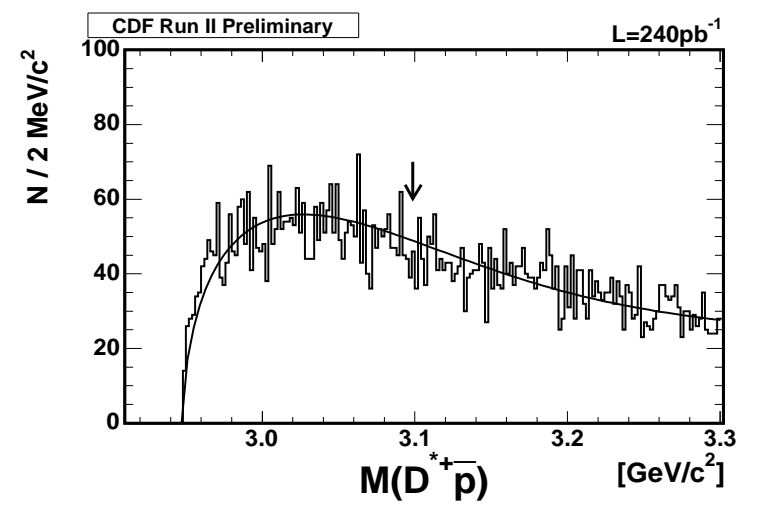

Figure 7. Mass of the $\left(D^{*+} \bar{p}\right)$ system. The arrow indicates the position of the $\Theta_{c}$ state $(\mathrm{CDF})$. 


\section{RECENT B PHYSICS RESULTS}

\subsection{Spectroscopy}

$\mathrm{CDF}$ has measured the mass of $b$ hadrons in exclusive $J / \psi$ channels. The measurements of the $B_{s}$ and $\Lambda_{b}$ (Fig. 8) masses are the current world's best.

$$
\begin{aligned}
& m\left(B^{+}\right)=5279.10 \pm 0.41(\text { stat }) \pm 0.36(\text { syst }), \\
& m\left(B^{0}\right)=5279.63 \pm 0.53(\text { stat }) \pm 0.33(\text { syst }), \\
& m\left(B_{s}\right)=5366.01 \pm 0.73(\text { stat }) \pm 0.33(\text { syst }), \\
& m\left(\Lambda_{b}\right)=5619.7 \pm 1.2(\text { stat }) \pm 1.2(\text { syst }) \mathrm{MeV} / c^{2} .
\end{aligned}
$$

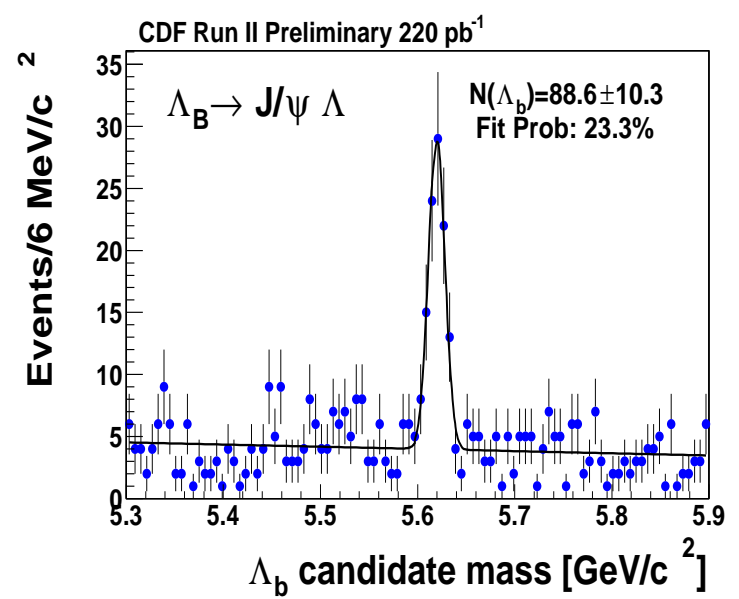

Figure 8. The mass spectrum of $\Lambda_{b}$ candidates $(\mathrm{CDF})$.

DØ reports the first observation of the excited $B$ mesons $B_{1}$ and $B_{2}^{*}$ as two separate states in fully reconstructed decays to $B^{(*)} \pi$. The mass of $B_{1}$ is measured to be $5724 \pm 4 \pm 7 \mathrm{MeV} / \mathrm{c}^{2}$, and the mass difference $\Delta M$ between $B_{2}^{*}$ and $B_{1}$ is $23.6 \pm 7.7 \pm 3.9 \mathrm{MeV} / \mathrm{c}^{2}$ (Fig. 9).

$\mathrm{D} \varnothing$ observes semileptonic $B$ decays to narrow $D^{* *}$ states, the orbitally excited states of the $D$ meson seen as resonances in the $D^{*+} \pi^{-}$invariant mass spectrum. The $D^{*}$ mesons are reconstructed through the decay sequence $D^{*+} \rightarrow$ $D^{0} \pi^{+}, D^{0} \rightarrow K^{-} \pi^{+}$. The invariant mass of oppositely charged $\left(D^{*}, \pi\right)$ pairs is shown in Fig. 10
The mass peak between 2.4 and $2.5 \mathrm{GeV} / c^{2}$ can be interpreted as two merged narrow $D^{* *}$ states, $D_{1}^{0}(2420)$ and $D_{2}^{0}(2460)$. The combined branching fraction is $\mathcal{B}\left(B \rightarrow D_{1}^{0}, D_{2}^{0}\right) \cdot \mathcal{B}\left(D_{1}^{0}, D_{2}^{0} \rightarrow\right.$ $\left.D^{*+} \pi^{-}\right)=(0.280 \pm 0.021$ (stat) \pm 0.088 (syst $) \%$. The systematic error includes the unknown phase between the two resonances. Work is in progress on extracting the two Breit-Wigner amplitudes.

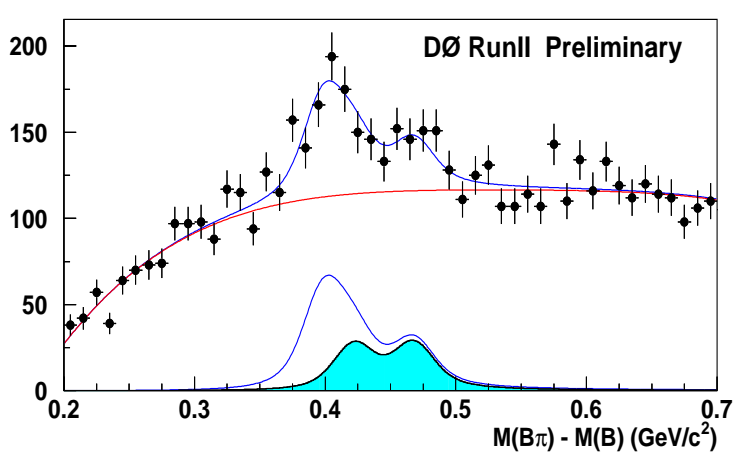

Figure 9. Mass difference $\Delta M=M(B \pi)-$ $M(B)$ for exclusive $B$ decays. The backgroundsubtracted signal is a sum of $B_{1}^{*} \rightarrow B^{*} \pi, B^{*} \rightarrow$ $B \gamma$ (open area) and $B_{2}^{*} \rightarrow B^{*} \pi B^{*} \rightarrow B \gamma$ (lower peak in the shaded area) and $B_{2}^{*} \rightarrow B \pi$ (upper peak in the shaded area) ( $\varnothing \varnothing)$.

\subsection{Lifetimes}

$\mathrm{CDF}$ and $\mathrm{D} \varnothing$ have measured lifetimes of $b$ hadrons through the exclusively reconstructed decays $B^{+} \rightarrow J / \psi K^{+}, B^{0} \rightarrow J / \psi K^{* 0}, B_{s} \rightarrow J / \psi \phi$, and $\Lambda_{b} \rightarrow J / \psi \Lambda$ (Fig. 11). The latest results are:

$$
\begin{aligned}
& \tau\left(B^{+}\right)=1.65 \pm 0.08{ }_{-0.123}^{+0.096} \mathrm{ps} \quad(\mathrm{D} \varnothing 2003) \text {, } \\
& \tau\left(B^{+}\right)=1.662 \pm 0.033 \pm 0.008 \mathrm{ps} \quad(\mathrm{CDF}) \text {, } \\
& \tau\left(B_{d}^{0}\right)=1.473_{-0.050}^{+0.052} \pm 0.023 \mathrm{ps} \text { (DØ). } \\
& \tau\left(B_{d}^{0}\right)=1.539 \pm 0.051 \pm 0.008 \text { ps }(\mathrm{CDF}) \text {, } \\
& \tau\left(B_{s}^{0}\right)=1.444_{-0.090}^{+0.098} \pm 0.020 \mathrm{ps}(\mathrm{D} \varnothing), \\
& \tau\left(B_{s}^{0}\right)=1.369 \pm 0.100 \pm{ }_{0.010}^{+0.008} \text { ps (CDF), } \\
& \tau\left(\Lambda_{b}\right)=1.221_{-0.179}^{+0.217} \pm 0.043 \text { ps (DØ), } \\
& \tau\left(\Lambda_{b}\right)=1.25 \pm 0.26 \pm 0.10 \text { ps (CDF 2003). }
\end{aligned}
$$




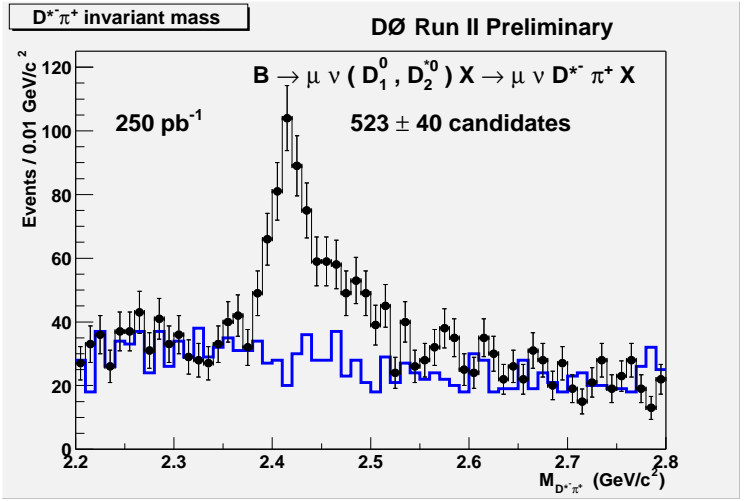

Figure 10. The invariant mass distribution of $\left(D^{*}, \pi\right)$ pairs, opposite sign (points) and samesign (solid histogram).

The measured lifetimes correspond to the following lifetime ratios:

$$
\begin{aligned}
& \tau\left(B^{+}\right) / \tau\left(B_{d}^{0}\right)=1.080 \pm 0.042(\mathrm{CDF}), \\
& \tau\left(B_{s}^{0}\right) / \tau\left(B_{d}^{0}\right)=0.890 \pm 0.072(\mathrm{CDF}), \\
& \tau\left(B_{s}^{0}\right) / \tau\left(B_{d}^{0}\right)=0.980_{-0.075}^{+0.075} \pm 0.003(\mathrm{D} \varnothing), \\
& \tau\left(\Lambda_{b}\right) / \tau\left(B_{d}^{0}\right)=0.874_{-0.142}^{+0.169} \pm 0.028(\mathrm{D} \varnothing) .
\end{aligned}
$$

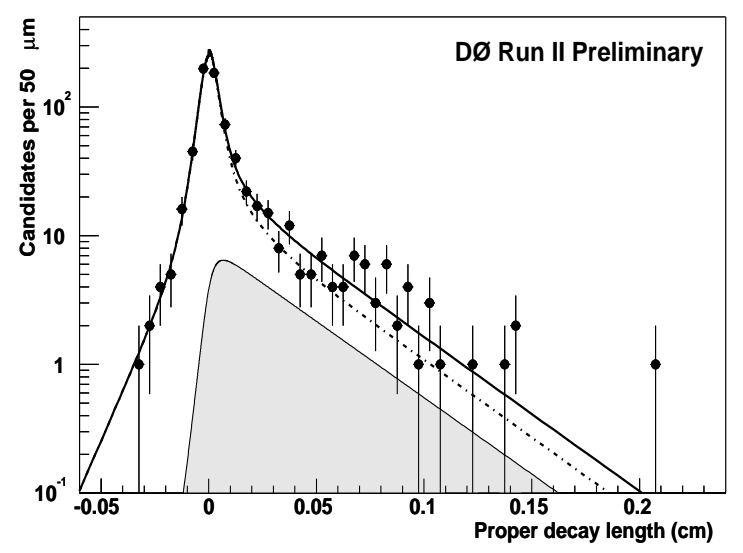

Figure 11. Fit projection on $c \tau$ for the $\Lambda_{b}$ candidates. (DØ)
The $B_{s}$ lifetime measurements listed above are results of a single-lifetime fit to data, integrated over the decay angles. Because of the presence of final states common to $B_{s}^{0}$ and its charge conjugate $\bar{B}_{s}^{0}$, the two meson states are expected to mix in such a way that the two CP eigenstates may have a relatively large lifetime difference. It is possible to separate the two $\mathrm{CP}$ components of $B_{s}^{0} \rightarrow J / \psi \phi$ and thus to measure the lifetime difference by studying the time evolution of the polarization states of the vector mesons in the final state. CDF has carried out a combined analysis of $B_{s}$ lifetimes and polarization amplitudes. The results for the lifetimes of the low mass (CP even) and high mass (CP odd) eigenstates, and the relative width difference are:

$$
\begin{aligned}
& \tau_{L}=1.05_{-0.13}^{+0.16} \pm 0.02 \mathrm{ps}, \\
& \tau_{H}=2.07_{-0.46}^{+0.58} \pm 0.03 \mathrm{ps}, \\
& \Delta \Gamma / \bar{\Gamma}=0.65_{-0.33}^{+0.25} \pm 0.01 .
\end{aligned}
$$

Figure 12 shows the scan of the likelihood function for $\Delta \Gamma / \bar{\Gamma}$. Pseudoexperiments tossed with $\Delta \Gamma / \bar{\Gamma}=0$ yield the betting odds for observing the above results at $1 / 315$. For $\Delta \Gamma / \bar{\Gamma}=0.12$ (SM prediction, which has recently been updated to $0.14 \pm 0.05[5]$ ) the betting odds are $1 / 84$.

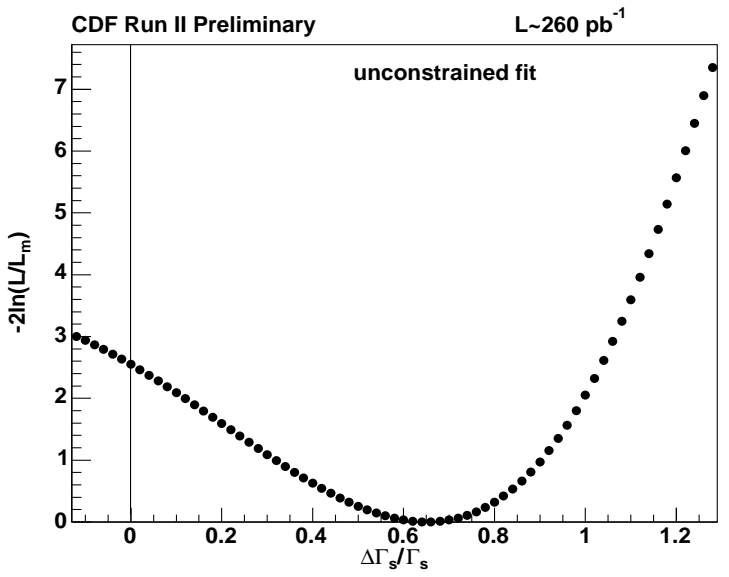

Figure 12. Scan of the likelihood function for $\Delta \Gamma / \bar{\Gamma}(\mathrm{CDF})$. 
$\mathrm{D} \varnothing$ has used a novel technique to measure the lifetime ratio of the charged and neutral $B$ mesons, exploiting the large semileptonic sample. $B$ hadrons were reconstructed in the channels $B \rightarrow \mu^{+} \nu D^{*}(2010)^{-} X$, which are dominated by $B^{0}$ decays, and $B \rightarrow \mu^{+} \nu D^{0} X$, which are dominated by $B^{+}$decays. The lifetime ratio was obtained from the variation of the ratio of the number of events in these two processes at different decay lengths. The result is

$$
\tau\left(B^{+}\right) / \tau\left(B_{d}^{0}\right)=1.093 \pm 0.021 \pm 0.022 .
$$

\subsection{Towards $B_{s}$ mixing}

Measurement of the $B_{s}$ oscillation frequency via $B_{s}^{0}-\bar{B}_{s}^{0}$ mixing will provide an important constraint on the CKM matrix. The oscillation frequency is proportional to the mass difference between the mass eigenstates, $\Delta m_{s}$, and is related to the CKM matrix through $\Delta m_{s} \propto\left|V_{t b} V_{t s}\right|$. When combined with the $B_{d}$ mass difference, $\Delta m_{d}$ it helps in extraction of $\left|V_{t d}\right|$, and thereby the $\mathrm{CP}$ violating phase.

As a benchmark for future $B_{s}$ oscillation measurement, both groups study $B_{d}$ mixing, gaining an understanding of the different components of a $B$ mixing analysis (sample composition, flavor tagging, vertexing, asymmetry fitting). For a sample of partially reconstructed decays $B \rightarrow$ $D^{*}(2010)^{+} \mu^{-} X$, DØ obtains $\Delta m_{d}=0.506 \pm$ $0.055($ stat $) \pm 0.049($ syst $)) \mathrm{ps}^{-1}$ and $\Delta m_{d}=$ $0.488 \pm 0.066$ (stat) \pm 0.044 (syst) $) \mathrm{ps}^{-1}$ when employing opposite side muon tagging and the same side tagging, respectively.

The CDF result for semileptonic channels is $\Delta m_{d}=0.536 \pm 0.037$ (stat) \pm 0.009 (s.c.) \pm 0.015 (syst) $\mathrm{ps}^{-1}$. CDF also reports a result on $B$ oscillations using fully reconstructed decays: $\Delta m_{d}=0.526 \pm 0.056$ (stat) \pm 0.005 (syst) $) \mathrm{ps}^{-1}$.

Reconstructing $B_{s}$ decays into different final states is another important step in the $B_{s^{-}}^{0}$ $\bar{B}_{s}^{0}$ mixing analysis. Thanks to the large muon and tracking coverage, $\mathrm{D} \emptyset$ is accumulating a high statistics sample of semileptonic $B_{s}$ decays. D reconstructs the $B_{s} \rightarrow D_{s}^{+} \mu^{-} X$ decays, with $D_{s}^{+} \rightarrow \phi \pi^{+}$and $D_{s}^{+} \rightarrow K^{*} K^{+}$, at a rate of $\approx$ $40(25)$ events per $\mathrm{pb}^{-1}$, respectively. Figure 13 shows the mass distribution of the $D_{s}^{+} \rightarrow \phi \pi$ can- didates.

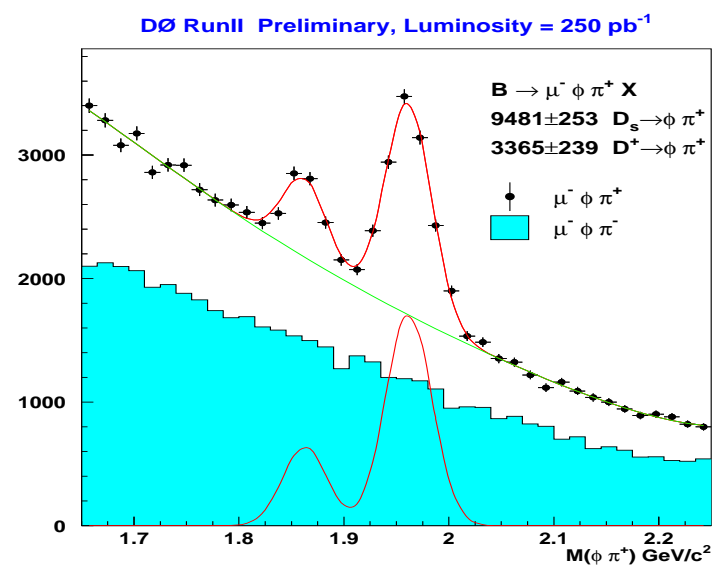

Figure 13. $D_{s}^{+} \rightarrow \phi \pi^{+}$signal. (DØ)

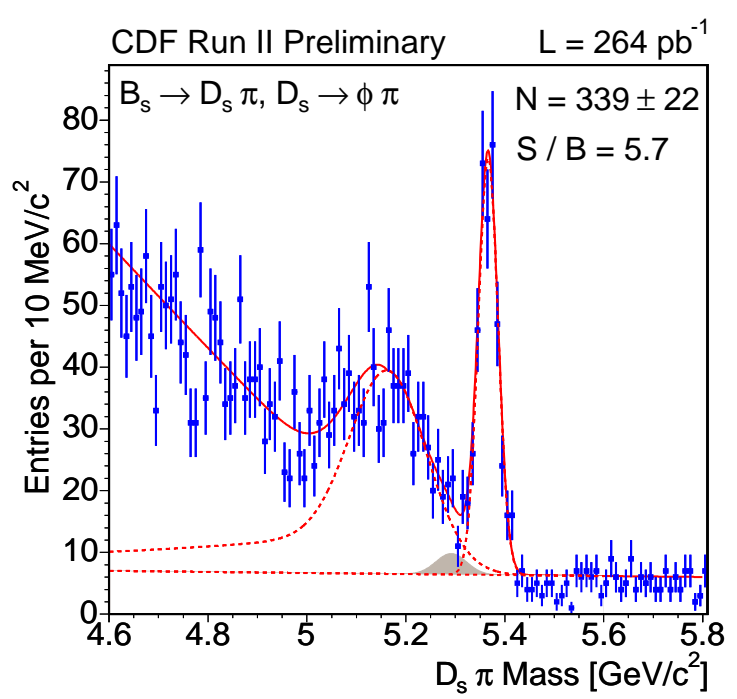

Figure 14. $B_{s} \rightarrow D_{s} \pi, D_{s} \rightarrow \phi \pi$ signal. (CDF)

CDF has clean signals for fully hadronic, flavorspecific $B_{s}$ decays, providing the best sensitivity to $B_{s}$ oscillations at high $\Delta m_{s}$. Figure 14 shows the signal for the best channel, $B_{s} \rightarrow D_{s} \pi, D_{s} \rightarrow$ $\phi \pi$. 


\subsection{Rare decays}

The purely leptonic decays $B_{d, s}^{0} \rightarrow \mu^{+} \mu^{-}$ are flavor-changing neutral current (FCNC) processes. In the standard model, these decays are forbidden at the tree level and proceed at a very low rate through higher-order diagrams. The latest SM prediction [6] is $\mathcal{B}\left(B_{s}^{0} \rightarrow \mu^{+} \mu^{-}\right)=$ $(3.42 \pm 0.54) \times 10^{-9}$, where the error is dominated by non-perturbative uncertainties. The leptonic branching fraction of the $B_{d}^{0}$ decay is suppressed by CKM matrix elements $\left|V_{t d} / V_{t s}\right|^{2}$ leading to a predicted SM branching fraction of $(1.00 \pm 0.14) \times 10^{-10}$. The best published experimental bound (Fig. 151) for the branching fraction of $B_{s}^{0}\left(B_{d}^{0}\right)$ is presently $\mathcal{B}\left(B_{s}^{0}\left(B_{d}^{0}\right) \rightarrow \mu^{+} \mu^{-}\right)<$ $7.5 \times 10^{-7}\left(1.9 \times 10^{-7}\right)$ at the $95 \%$ C.L. 7. The decay amplitude of $B_{d, s}^{0} \rightarrow \mu^{+} \mu^{-}$can be significantly enhanced in some extensions of the SM.

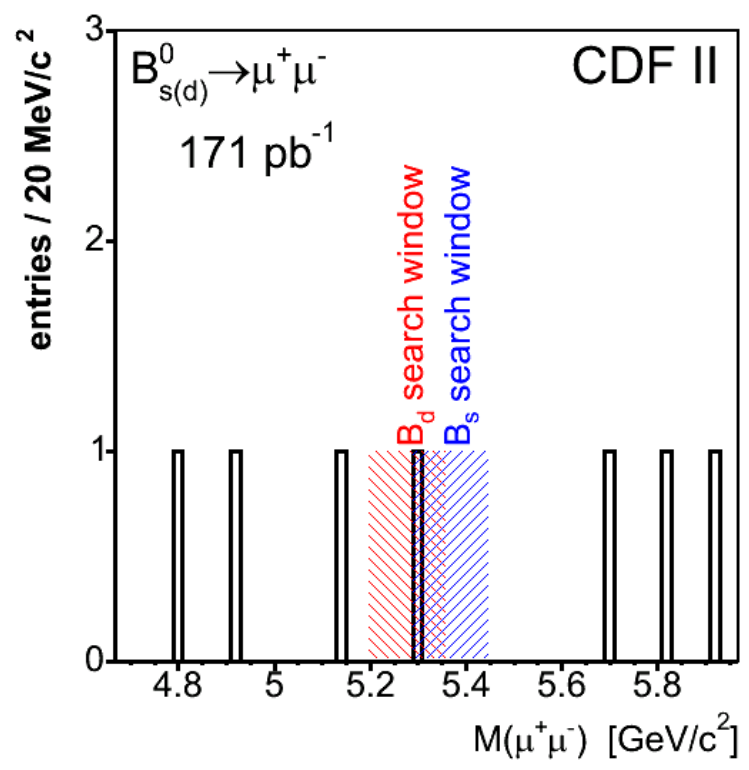

Figure 15. Invariant mass for the events passing all requirements. (CDF)

Assuming no contributions from the decay $B_{d}^{0} \rightarrow \mu^{+} \mu^{-}$in the signal region, $\mathrm{D} \varnothing$ finds the conservative upper limit on the branching frac- tion to be $\mathcal{B}\left(B_{s}^{0} \rightarrow \mu^{+} \mu^{-}\right) \leq 4.6 \times 10^{-7}$ at the 95\% C.L. (Fig. 16).

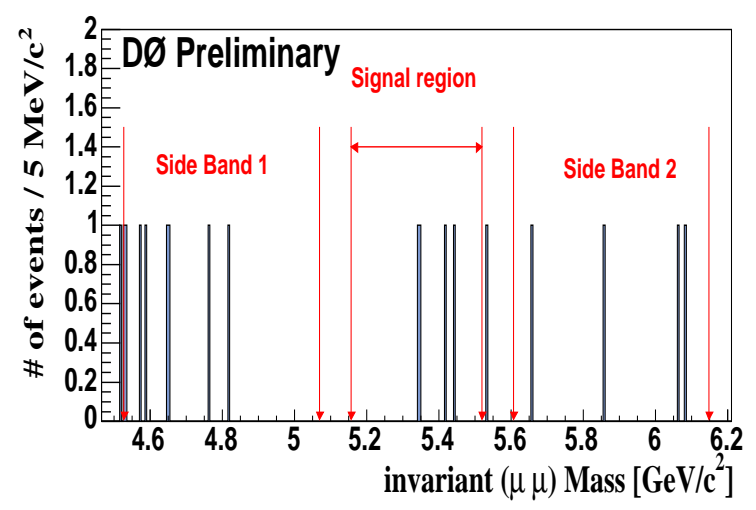

Figure 16. Invariant mass for the events passing all requirements. $(\mathrm{D} \varnothing)$

\section{REFERENCES}

1. V. M. Abazov et al. DØ Collaboration, Nature 429 (2004). 638.

2. S.K. Choi et al. (Belle Collaboration), Phys. Rev. Lett. 91, 262001 (2003).

3. D. Acosta et al. (CDF Collaboration), Phys. Rev. Lett. 93, 072001 (2004).

4. V. M. Abazov et al. (DØ Collaboration), submitted to Phys. Rev. Lett.

5. U. Nierste, FNAL Chicago Flavor seminar, July 2004.

6. A. J. Buras, Phys. Lett. B 566, 115 (2003).

7. D. Acosta et al. (CDF Collaboration), Phys. Rev. Lett. 93, 032001 (2004). 